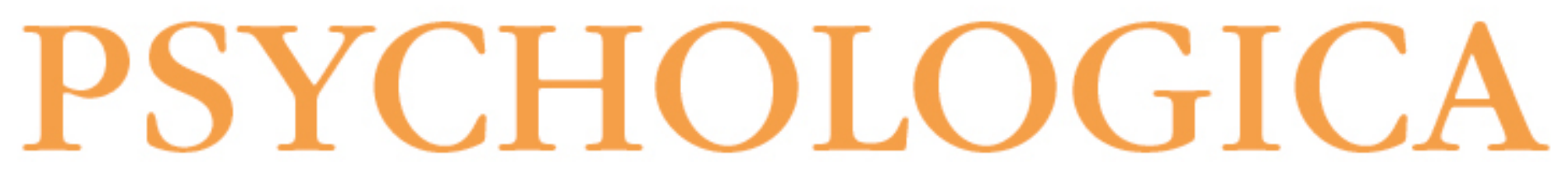

Factores de adaptação e recuperação após uma doença hemato-oncológica: um estudo exploratório

Autor(es): $\quad$ Reis, Sílvia; Narciso, Isabel

Publicado por: Imprensa da Universidade de Coimbra

URL

persistente: URI:http://hdl.handle.net/10316.2/3453

DOI: DOl:http://dx.doi.org/10.14195/1647-8606_52-1_16

Accessed : $\quad$ 26-Apr-2023 11:22:34

A navegação consulta e descarregamento dos títulos inseridos nas Bibliotecas Digitais UC Digitalis, UC Pombalina e UC Impactum, pressupõem a aceitação plena e sem reservas dos Termos e Condições de Uso destas Bibliotecas Digitais, disponíveis em https://digitalis.uc.pt/pt-pt/termos.

Conforme exposto nos referidos Termos e Condições de Uso, o descarregamento de títulos de acesso restrito requer uma licença válida de autorização devendo o utilizador aceder ao(s) documento(s) a partir de um endereço de IP da instituição detentora da supramencionada licença.

Ao utilizador é apenas permitido o descarregamento para uso pessoal, pelo que o emprego do(s) título(s) descarregado(s) para outro fim, designadamente comercial, carece de autorização do respetivo autor ou editor da obra.

Na medida em que todas as obras da UC Digitalis se encontram protegidas pelo Código do Direito de Autor e Direitos Conexos e demais legislação aplicável, toda a cópia, parcial ou total, deste documento, nos casos em que é legalmente admitida, deverá conter ou fazer-se acompanhar por este aviso.

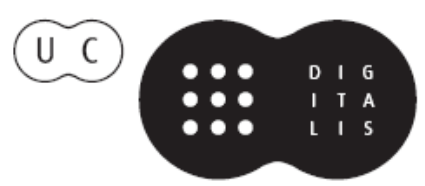




\section{NÚMERO 52}

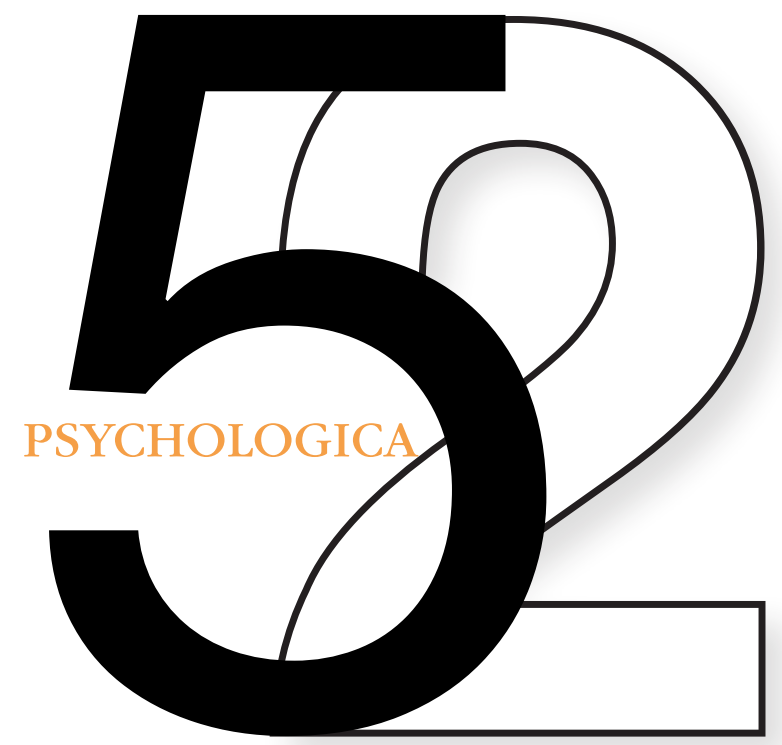

VOLUME I

IMPRENSA DA UNIVERSIDADE DE COIMBRA

FACULDADE DE PSICOLOGIA E DE CIÊNCIAS DA EDUCAÇÃO DA UNIVERSIDADE DE COIMBRA 


\title{
Factores de adaptação e recuperação após uma doença hemato-oncológica - um estudo exploratório
}

\author{
Sílvia Reis' \& Isabel Narciso²
}

Pretendemos analisar factores que podem favorecer ou dificultar o processo de adaptação em indivíduos com uma doença do foro hemato-oncológico. A recolha de dados, com uma amostra de 101 sujeitos em remissão de doença hemato-oncológica, incluiu um questionário sócio-biográfico, o Questionário de Orientação para Viver (QOV- Antonovsky, 1998b), o Questionário de Bem-Estar Psicológico (BEP- Ryff, 1989), o Questionário da Qualidade de Vida (EORTC OLLQ-C30 - versão 3-Aaronson et al., 1993) e, o Questionário da Qualidade das Relações Familiares (FES- Moos, 1990). Os resultados obtidos permitem-nos constatar que os sujeitos com nível de escolaridade mais baixo percepcionam-se menos capazes de se adaptarem ao stress; o auto-transplante de medula óssea é um factor de stress adicional e significativo; o diagnóstico de Mieloma múltiplo parece ser indutor de maior stress; os mais jovens, os não casados, os com maior nível de ensino, os empregados e os que não têm filhos têm maior predisposição para uma melhor recuperação; e, o clima relacional familiar constitui um recurso que poderá contribuir para níveis menos elevados de stress e utilização de estratégias mais adaptativas.

PALAVRAS-CHAVE: Doenças hemato-oncológicas, sentido de coerência, bem-estar psicológico, qualidade de vida, clima relacional familiar.

\section{Enquadramento conceptual}

\subsection{Doenças hemato-oncológicas}

Apesar de um aumento da probabilidade dos indivíduos sobreviverem cinco ou mais anos após o diagnóstico de cancro, Ribeiro (1998) diz-nos que o cancro é a segunda causa de morte, nos adultos, nos países do mundo ocidental. As doenças hemato-oncológicas não são excepção. Entre estas, as que apresentam maior taxa

\footnotetext{
1 Licenciada em Enfermagem pela Escola Superior de Enfermagem Dr. Ângelo da Fonseca. Mestre em Ciências da Educação - Aprendizagem e Desenvolvimento do Adulto e do Idoso, pela Faculdade de Psicologia e de Ciências da Educação da Universidade de Lisboa. - smagda@portugalmail.com

2 Professora Auxiliar da Faculdade de Psicologia e de Ciências da Educação da Universidade de Lisboa. - narciso@fpce.ul.pt
} 
de incidência dividem-se em três grupos: Mieloma múltiplo, Linfoma e Leucemia. Os Linfomas subdividem-se em Hodgkin ou Não Hodgkin. Por sua vez, as Leucemias dividem-se em crónicas ou agudas e cada uma destas em mielóide ou linfóide. As Leucemias agudas podem ainda ser bifenotípicas. O tratamento pode assentar em quatro linhas base: quimioterapia, radioterapia, transplante de medula óssea e cirurgia. Cada tratamento varia de indivíduo para indivíduo, de acordo com a presença de determinados factores, entre eles, idade do doente, estádio do tumor, o número de sítios extranodais que apresentam doença, o estado clínico geral do doente, e se este se encontra pela primeira vez a fazer quimioterapia ou se é precedente de uma recidiva.

\subsection{Perspectiva salutogénica}

Durante várias décadas, os estudos em Psicologia centraram-se, sobretudo, no negativo e na desadaptação. Contudo, esta tendência tem-se vindo a inverter, realçando-se a importância da compreensão do positivo e adaptativo para um maior enriquecimento da prevenção e intervenção. Assim, nas últimas décadas do século XX, foi introduzido, por Antonovsky (1998a,b), o modelo salutogénico como um novo paradigma para a investigação em saúde psicológica, enfatizando-se conceitos como sentido de coerência, resiliência, bem-estar psicológico e qualidade de vida.

No entender de Walsh (1998), resiliência pode ser definida como a capacidade para ultrapassar a adversidade com mais força e recursos, através de um processo activo de resistência, auto-reparação e crescimento, em resposta à crise e à mudança. É um conceito que implica muito mais que sobrevivência ou "passar por cima" de determinada situação. Os sobreviventes não são necessariamente resilientes, uma vez que alguns isolam-se em posições de vítimas impedidas de crescer, inundados por sentimentos de culpa e de fúria. Os indivíduos resilientes são capazes de lidar com situações geradoras de sofrimento, tomar conta das suas próprias vidas e seguir em frente com elas; ou seja, a resiliência resulta do confronto com a adversidade e não a fuga em relação a ela.

Antonovsky propõe o construto "sentido de coerência", para explicar como alguns indivíduos, apesar das contrariedades, vêem o mundo como ordenado, estruturado e com sentido, de forma a mobilizarem os recursos necessários à adaptação (Antonovsky, 1998a). Para este autor, o sentido de coerência (SCO) é conceptualizado como uma orientação global com a qual um indivíduo, com um contínuo e dinâmico sentimento de confiança, considera: (1) os estímulos resultantes do meio interno ou externo ao longo do curso de vida, como estruturados, preditiveis e explicáveis (capacidade de compreensão - comprehensibility); (2) que existem, ao seu alcance, recursos para satisfazer as exigências colocadas por 
esses estímulos (capacidade de gestão - manageability); e (3) que essas exigências constituem desafios merecedores de investimento e empenho do indivíduo (capacidade de investimento - meaningfulness) (Antonovsky, 1987, referenciado por Antonovsky, 1998b).

O bem-estar é uma das orientações mais dinâmicas da corrente da psicologia positiva, assumindo uma importância crescente nas últimas décadas. Esta corrente da psicologia preocupa-se mais em compreender o que contribui para uma vida feliz dos indivíduos, do que em corrigir as patologias que os afectam, ou seja, tem como alvo construir qualidades positivas e mostrar as acções que conduzem ao bem-estar (Seligman \& Csikszentmihalyi, 2000). Na década de 1980, Ryff propõe o modelo de bem-estar psicológico que emerge de diferentes modelos teóricos da psicologia do desenvolvimento, da psicologia clínica e da saúde mental. Estes diferentes modelos deram origem a seis dimensões caracterizadoras do bem-estar psicológico (BEP): aceitação de si, relações positivas com os outros, autonomia, domínio do meio, objectivos na vida e crescimento pessoal (Ryff, 1989; Novo, 2003).

A qualidade de vida (QDV) é um conceito relativamente recente. Em ambientes de saúde, é frequentemente referenciada como qualidade de vida relacionada com a saúde (ou seja, que está dependente da doença que o indivíduo tem), ou simplesmente como qualidade de vida (Ribeiro, 2001, 2006). A Organização Mundial de Saúde definiu qualidade de vida como sendo a percepção individual da sua posição na vida, no contexto da sua cultura e do seu sistema de valores, tendo em consideração os seus objectivos, expectativas, padrões e preocupações (World Health Organization, 1997). Também Haas (1999) e Santos e Ribeiro (2001) referem que se trata de uma avaliação individual das circunstâncias de vida e, de um conceito complexo, multidimencional, dinâmico (alterando-se de acordo com o tempo e as situações vivenciais), com componentes objectivas (funcionalidade, competência cognitiva e interacção com o meio) e subjectivas (sentimentos de satisfação e percepção da sua saúde).

Sendo a família, regra geral, o ponto de apoio mais próximo e mais significativo do indivíduo, pensamos que a qualidade das relações familiares, ou a percepção sobre elas, será fundamental no processo de adaptação e recuperação. Em situações de grande adversidade, como, por exemplo, no caso de doenças crónicas graves, a coesão familiar, a expressão de afectos, a partilha de sentimentos e um baixo nível de conflitos, são condições essenciais para o processo de adaptação e recuperação do equilíbrio individual e familiar (Pereira \& Lopes, 2005; Weihs \& Politi, 2005). É, ainda, importante considerar que a doença crónica num membro pode provocar efeitos profundos no desenvolvimento de outro membro, e, ainda, que os diferentes membros não se adaptam de forma uniforme à doença (Rolland, 1989). 


\section{O Presente Estudo}

Partindo de uma interrogação mais geral sobre a natureza e o papel de alguns factores de adaptação e recuperação após uma doença hemato-oncológica, e atendendo a uma perspectiva de complexidade sistémica, definimos como objectivos analisar, também em função de diversas variáveis demográficas e de saúde física, a percepção do sentido de coerência, a percepção do bem-estar psicológico, a percepção da qualidade de vida, a percepção da qualidade das relações familiares e a relação entre tais variáveis, nos indivíduos que experienciaram uma doença do foro hemato-oncológico.

No estudo realizado, partimos de algumas hipóteses: (1) nos sujeitos sobreviventes a uma doença hemato-oncológica, o bem-estar psicológico e a qualidade de vida estão associados ao tempo pós-transplante e ao tempo de remissão; (2) os sujeitos sobreviventes de Linfoma apresentam um sentido de coerência mais elevado que os sobreviventes a Leucemia; (3) um baixo sentido de coerência está associado a baixa qualidade de vida; (4) os sujeitos a quem foi diagnosticado Linfoma, apresentam níveis superiores de funcionalidade (de papel e social) e valores inferiores de sintomas físicos, que aos sujeitos a quem foi diagnosticado Leucemia ou Mieloma múltiplo; (5) o bem-estar psicológico e a qualidade de vida estão associados ao sentido de coerência; (6) os sujeitos não submetidos a auto-transplante, em comparação com os sujeitos auto-transplantados, têm níveis superiores de bem-estar psicológico e de qualidade de vida superiores; (7) o sentido de coerência é idêntico em sujeitos auto-transplantados e sujeitos não submetidos a auto-transplante; (8) a qualidade das relações familiares está associada ao sentido de coerência; (9) o bem-estar psicológico e a qualidade de vida estão associados ao sentido de coerência e à qualidade das relações familiares.

\section{Método}

\subsection{Amostra}

A amostra é constituída por 101 sujeitos: sobreviventes a Linfoma (66 sujeitos $(65,3 \%))$, Leucemia (16 sujeitos $(15,8 \%))$ ou Mieloma múltiplo (19 sujeitos $(18,8 \%)$ ); de ambos os sexos ( 63 homens $(62,4 \%$ ) e 38 mulheres $(37,6 \%)$ ); com idades compreendidas entre os 20 e os 64 anos ( 50 adultos com idades entre os 20 e os 44 anos (49,5\%) e 51 adultos com idades entre os 45 e os 64 anos (50,5\%)); 75 sujeitos casados ou em união de facto $(74,3 \%)$ e 26 sujeitos não casados $(25,7 \%) ; 62$ sujeitos com nível de ensino desde a primária até ao $3^{\circ}$ ciclo (61,5\%) e 39 sujeitos com o secundário ou o ensino superior (38,5\%); 48 sujeitos empregados (47,5\%) e 53 sujeitos não empregados (52,5\%); 73 sujeitos com filhos $(72,9 \%)$ e 28 sujeitos 
sem filhos (27,7\%); 50 sujeitos foram submetidos a auto-transplante de medula óssea (49,5\%) e 51 sujeitos não foram submetidos (50,5\%); 35 sujeitos com tempo de transplante até 24 meses $(34,7 \%)$ e 15 sujeitos com tempo de transplante de 25 a 60 meses (14,9\%); 28 sujeitos com tempo de remissão até 24 meses $(27,7 \%)$ e 23 sujeitos com tempo de remissão de 25 a 60 meses (22,8\%).

\subsection{Instrumentos de recolha de dados}

A recolha dos dados foi efectuada através de um protocolo com todos os instrumentos que, a seguir se descrevem, e, ainda, um Questionário Geral sobre dados sócio-demográficos e de saúde física, na Consulta Externa do Serviço de Hematologia Clínica, dos Hospitais da Universidade de Coimbra.

\section{Sentido de Coerência}

O sentido de Coerência foi avaliado através do Questionário de Orientação para Viver (OOV; Antonovsky, 1998b), com 29 itens respondidos numa escala de Likert de 1 a 7 . É constituído, originalmente, por três sub-escalas: capacidade de gestão, de compreensão e de investimento. No entanto, após análise factorial realizada no âmbito do nosso estudo (resultando na eliminação de 10 itens), obtivemos 2 factores - Avaliação e Expectativas sobre a Realidade, e Percepção de Organização e Coerência -, os quais foram avaliados, neste estudo, conjuntamente com o Sentido de Coerência Global. Relativamente à fidelidade, os valores de alpha encontrados são considerados bons, variando entre .87 e .75 .

\section{Bem-Estar Psicológico}

O Bem-Estar Psicológico foi avaliado através da Escala de Bem-Estar psicológico (Ryff, 1989; Novo, 2003), constituída por 18 itens, respondidos numa escala de Likert de 1 a 6. Esta escala é composta por 6 sub-escalas - Aceitação de Si, Relação com os Outros, Autonomia, Domínio do Meio, Objectivos na Vida, Crescimento Pessoal -, as quais foram avaliadas para a população estudada conjuntamente com o BEP global. Quanto aos valores de alpha de Cronbach das seis sub-escalas do BEP, apresentam-se baixos, variando entre .38 e .68. No entanto, o valor de alpha de BEP global pode-se considerar bom (.85).

\section{Qualidade de Vida}

Para avaliar a Qualidade de Vida, utilizou-se o Questionário de Qualidade de Vida (EORTC OLQ-C30 versão 3; Aaronson et al., 1993), com 30 itens, uns de resposta dicotómica sim-não, e outros em escala de Likert de 1 a 4 ou de 1 a 7. Apresenta 
uma estrutura de cinco escalas de funcionalidade (física, de papel, emocional, cognitiva e social); três escalas de sintomas (fadiga, dor e náusea e vómito); uma escala de avaliação de saúde e qualidade de vida globais; e, ainda, seis itens únicos de avaliação de sintomas (dispneia, insónia, perda de apetite, prisão de ventre, diarreia e dificuldades financeiras). Os valores de alpha de Cronbach do total do questionário de Qualidade de Vida e das suas sub-escalas são considerados bons, variando entre .65 e .91 .

\section{Clima Relacional Familiar}

A avaliação do clima relacional familiar foi efectuada através da Escala da Qualidade das Relações Familiares (FES; Moos, 1990), tendo sido apenas utilizadas as três sub-escalas da dimensão Relação - Coesão, Expressividade e Conflito -, num total de 27 itens. Em Portugal, este instrumento foi traduzido e adaptado para a população portuguesa por Matos e Fontaine (1992, referenciados por Moura, 2005), sendo cada item avaliado numa escala de Likert com seis categorias de resposta. Apesar de termos realizado todas as operações estatísticas no sentido de adaptar a escala à nossa amostra, optámos por utilizá-la apenas numa perspectiva de análise sobretudo qualitativa, uma vez que encontrámos várias incoerências conceptuais durante o processo de análise factorial. Assim, optámos por uma análise percentual dos valores da escala de Likert, para cada item da escala, o que nos permite avaliar o clima relacional familiar, enquanto recurso, identificando zonas de "força" e de "fragilidade" relativamente à coesão, expressividade e conflito.

\section{Apresentação e Discussão dos Resultados}

\subsection{Sentido de Coerência}

Pela análise dos dados, concluímos, através do teste paramétrico Independent Sample Test, para todas as variáveis com excepção da variável diagnóstico onde foi utilizado o teste paramétrico Oneway Anova, que se encontram valores médios superiores de Sentido de Coerência Global nos sujeitos com maior nível de ensino (secundário e superior: $M=91.15$ vs Primário, $2^{\circ}$ e $3^{\circ}$ ciclos: $M=82.32, p=.008$ ) e nos que não realizaram auto-transplante de medula óssea (Sem transplante: $M=90.33$ vs Com transplante: $M=81.04, p=.004)$. Relativamente a Avaliação e Expectativas sobre a Realidade, os resultados são também significativamente superiores nos participantes com maior escolaridade ( $M=58.19$ vs $M=50.13, p=.004)$ e que não realizaram auto-transplante $(M=57.06$ vs $M=49.28, p=.004)$. São, também, os sujeitos a quem foi diagnosticado Linfoma ou Leucemia que apresentam valores médios superiores de Avaliação e Expectativas sobre a Realidade (Leuc.: $M=57.44$, Linf.: $M=54.36$ vs Mieloma: $M=45.63, p=.021$ ). 
Relativamente à variável nível de ensino frequentado, a literatura revela inconsistência de resultados. Assim, os estudos realizados por Nilsson, Holmgren e Westman (2000) e por Holmberg, Thelin e Stiernstrom (2004) referem não existir relação entre o nível educacional e o SCO. Por outro lado, os estudos realizados por Callahan e Pincus (1995, referenciados por Holmberg, Thelin \& Stiernstrom, 2004), por Svartvik, Lidfeldt, Nerbrand, Samsioe, Schersten e Nilsson (2000), e por Richardson et al. (2001, referenciados por Holmberg, Thelin \& Stiernstrom, 2004), apresentaram uma correlação positiva entre o SCO e o nível educacional, resultados estes corroborados pelo nosso estudo.

A nível da realização de auto-transplante de medula óssea, os resultados refutam a nossa $7^{\text {a }}$ hipótese. A revisão bibliográfica efectuada não revelou estudos que diferenciem valores de sentido de coerência, em indivíduos expostos a diferentes factores de stress. No entanto, Antonovsky (1998a) refere que todas as estruturas e contextos que envolvem o individuo influenciam a natureza dos factores de stress que enfrenta, e moldam a intensidade e o desenvolvimento do sentido de coerência individual (incluindo, aqui, as experiências familiares, o contexto familiar relacional e todos os contextos onde o indivíduo e a família se inserem). O aparecimento de uma doença hemato-oncológica é considerado um acontecimento de vida não normativo. Será suficientemente significativo ao ponto de implicar mudanças na forma de ver o mundo, de lhe dar sentido e de mobilizar os recursos necessários à adaptação? É certo que os dois grupos de sujeitos vivenciaram, e ainda vivenciam, acontecimentos de vida muito stressantes. No entanto, os sujeitos submetidos a auto-transplante de medula óssea experienciaram um factor de stress adicional. Pela análise dos nossos resultados, parece ser este factor de stress adicional (auto-transplante de medula óssea) o responsável pela diferenciação, nos dois grupos de sujeitos estudados, dos valores médios do sentido de coerência global e de uma das suas dimensões - Avaliação e Expectativas sobre a Realidade.

No que diz respeito à variável diagnóstico, uma explicação plausível para as diferenças encontradas talvez se prenda com o facto de o Mieloma múltiplo não ter cura, o que significa que os sujeitos, a quem foi diagnosticada esta patologia, não alcançam a remissão completa, apenas remissões parciais. Desta forma, é compreensivel que este grupo de sujeitos apresente valores inferiores na avaliação do mundo e do próprio e nas expectativas sobre o próprio e sobre os outros. Os nossos resultados revogam a hipótese 2 , por nós proposta, o que nos leva a pensar que os baixos níveis de stress apresentados pelos sobreviventes à doença Linfoma de Hodgkin e os altos níveis de stress apresentados pelos sobreviventes à doença Leucemia, encontrados por Wettergren, Langius, Bjorkholm \& Bjorvell (1999), e Kornblith, et al. (1992), não são suficientes para causar diferenças no sentido de coerência dos sujeitos a quem foram diagnosticadas estas patologias. 


\subsection{Bem-Estar Psicológico}

Os resultados (através do teste paramétrico Independent Sample Test para o BEP global e para as dimensões relações positivas com os outros, domínio do meio e autonomia, e do teste não paramétrico de Mann-Whitney para as dimensões aceitação de si, crescimento pessoal e objectivos na vida) parecem indicar valores médios superiores de bem-estar psicológico global e/ou em pelo menos uma das suas sub-escalas nos sujeitos mais jovens (BEP Global - 20-44: $M=85.12$ vs 45-64: $M=76.27, p=.001$; Relações Positivas $-20-44: M=13.58$ vs 45-64: $M=11.84, p=.013$; Autonomia - 20-44: $M=13.20$ vs 45-64: $M=11.31, p=.002$; Crescimento Pessoal - 20-44: $M=61.93$ vs 45-64: $M=40.28, p=.000$; Objectivos na Vida $-20-44: M=59.35$ vs 45-64: $M=42.81, p=.004$ ), os não casados (Crescimento Pessoal - Não casados: $M=65.92$ vs Casados: $M=45.83, p=.002$ ), os com maior nível de ensino (BEP Global -Secund. e Superior: $M=86.77$ vs Até $3^{\circ}$ Ciclo: $M=76.81, p=.000$; Relações Positivas - Secund. e Superior: $M=14.46$ vs Até $3^{\circ}$ Ciclo: $M=11.60, p=.000$; Autonomia - Secund. e Superior: $M=13$ vs Até $3^{\circ}$ Ciclo: $M=11.77, p=.045$; Crescimento Pessoal - Secund. e Superior: $M=67.53$ vs Até $3^{\circ}$ Ciclo: $M=40.60, p=.000$; Objectivos na Vida - Secund. e Superior: $M=64.95$ vs Até $3^{\circ}$ Ciclo: $M=42.23, p=.000$ ), os empregados (Objectivos na Vida - Empregados: $M=58.80$ vs Não Empregados: $M=43.93, p=010)$, os que vivem sem filhos (Autonomia - Sem Filhos: $M=13,25$ vs Com Filhos: $M=11.86, p=.023$; Crescimento Pessoal - Sem Filhos: $M=67.13$ vs Com Filhos: $M=44.82, p=.001$ ), e a quem foi diagnosticado Leucemia (Bem-Estar Global $-M=83.63, p=.033$; Autonomia $-M=64.25, p=.010$ ) ou Linfoma (Objectivos de Vida $-M=54.95, p=.048$ ).

Os nossos resultados revelam que os sujeitos mais jovens apresentam valores mais elevados de bem-estar psicológico global, de relações positivas com os outros, de autonomia, de crescimento pessoal e de objectivos na vida. Estes resultados vão ao encontro das teorias do desenvolvimento, visto que, segundo vários autores (Marchand, 2001; Levinson, 1990 e Erikson, 1950/76, referenciado por Marchand, 2001), este é um período de grande energia e expansão, com o assumir de novos papéis familiares e sociais, e com a diminuição do apoio e autoridade parental. Já nos sujeitos mais velhos, parece haver uma atitude de desistência perante os acontecimentos de vida menos positivos, numa fase do ciclo de vida que se caracteriza como a mais criativa, onde os indivíduos se sentem menos tiranizados pelas paixões, ambições e ilusões da juventude, (Marchand, 2001); onde os indivíduos se sentem menos tiranizados pelos conflitos internos e exigências externas (Levinson, 1990); e, onde procuram contribuir para o bem-estar presente e futuro de outras gerações (Erikson, 1950/76, referenciado por Marchand, 2001). Resultados de investigações anteriores (Novo, Silva \& Peralta, 1997, referenciadas por Novo, 2003; Novo, 2003; Ryff, 1989; Ryff, 1991; Ryff \& Keyes, 1995) divergem dos nossos. Este facto pode estar relacionado com: o cancro aumentar o sentido de alienação face à vida familiar e a perda de controlo das circunstâncias da vida, 
tal como é referido por Krailik (2002); a grande variabilidade do grupo etário dos 45 aos 64 anos; e, os grupos etários que estudámos não corresponderem às faixas etárias das investigações referidas.

Relativamente à variável nível de ensino frequentado, os resultados indicam que uma maior literacia é preditora do funcionamento psicológico positivo, nomeadamente no campo das relações positivas com os outros, da autonomia, do crescimento pessoal e dos objectivos na vida. O ensino, para além de ensinar competências técnicas, abrange outras esferas do desenvolvimento, nomeadamente a social, a relacional e a psicológica.

Os sujeitos empregados apresentam valores superiores na dimensão objectivos na vida. Pensamos que estes resultados poderão ter duas interpretações possíveis. Por um lado, o facto de que estar empregado, após ter vivenciado uma doença hemato-oncológica, signifique estar livre da doença e com poucas sequelas (pelo menos que não os impeça de desenvolver uma actividade profissional), levando-os a dar significado e direcção às vivências passadas e presentes, e estabelecer mais objectivos e metas futuras. Tal como defendem Gotay, Isaacs e Pagano (2004, referenciados por Boyle, 2006), após passar por uma experiência de ameaça de vida, como é o cancro, os indivíduos tendem a reexaminar o sentido da vida e reconfigurar as medidas pessoais de qualidade de vida. Por outro lado, estes objectivos e metas podem ser profissionais, já que a única característica diferenciadora dos dois grupos de sujeitos é que uns estão empregados e os outros não.

Ao analisar a variável estado civil, constatamos a existência de diferenças significativas apenas para a dimensão crescimento pessoal, sendo os sujeitos não casados a apresentar os valores mais elevados. A bibliografia revista, contraria parcialmente os nossos resultados. Ryff (1989) referencia que ser casado é preditor de aceitação de si e de objectivos na vida, levando-nos a concluir que estas duas dimensões apresentam valores superiores para os casados, em relação a qualquer outro estado civil. Também Baider, Koch, Esacson e De-Nour (1998), evidenciam que os indivíduos casados apresentam um nível de bem-estar físico e mental mais elevado, maior habilidade para enfrentar a doença, melhor compreensão dos eventos ameaçadores, maior motivação para novas acções e menor stress emocional.

Revendo os resultados encontrados para a variável existência de filhos, verificamos, também, que as dimensões autonomia e crescimento pessoal apresentam valores significativamente mais baixos para quem tem filhos. Pensamos que, se por um lado, a família e os filhos podem contribuir positivamente para o crescimento pessoal, tal como também referem Walsh (1998) e Riley e Masten (2005), por outro lado, a vivência de uma doença como o cancro em que a perspectiva de morte é uma forte e real possibilidade e, a incerteza quanto à continuidade 
de uma vida familiar ou a antecipação de "perda" da família e dos filhos, pode conduzir à estagnação pessoal, e à falta de interesse pela vida.

Relativamente à variável diagnóstico, os resultados revelam que os sujeitos a quem foi diagnosticado Leucemia, apresentam valores superiores de bem-estar psicológico global e de crescimento pessoal, quando comparados com os sujeitos a quem foi diagnosticado Mieloma múltiplo. Por outro lado, os sujeitos a quem foi diagnosticado Linfoma, apresentam valores superiores de objectivos na vida, quando comparados com os sujeitos a quem foi diagnosticado Mieloma múltiplo. Uma explicação plausível para as diferenças encontradas talvez se prenda com o facto de o Mieloma múltiplo não ter cura, o que significa que os sujeitos, a quem foi diagnosticada esta patologia, não alcançam a remissão completa, apenas remissões parciais.

\subsection{Qualidade de vida}

Pela análise da Tabela I, concluímos que as variáveis idade, estado civil, nível de ensino frequentado, profissão, e existência de filhos apresentam valores significativamente diferentes em, pelo menos, uma das escalas ou itens que avaliam a qualidade de vida. Relativamente ao tempo de transplante, apenas se encontraram valores significativamente superiores na variável Dificuldades Financeiras para um tempo de transplante até 24 meses comparativamente com 25 a 60 meses $(M=28.13, p=.039)$. Também no tempo de remissão até 24 meses se encontram valores significativamente superiores no que se refere à variável Função Social $(M=30.30, p=.015)$

Tabela I. Valores de p e média da Qualidade de Vida em função da idade, do estado civil, do nivel de ensino, da profissão e da existência de filhos.

\begin{tabular}{|c|c|c|c|c|c|c|c|c|}
\hline & F. físico & F. papel & F. cogniti & F. social & SOQVG & Fadiga & Dor & Dif. Financ \\
\hline Idade: & $p=, 000$ & $p=, 003$ & $p=, 003$ & \multirow{3}{*}{ n.s. } & $p=, 000$ & $p=, 007$ & $p=, 025$ & \multirow{3}{*}{ n.s. } \\
\hline 20 a $44 \mathrm{~A}$ & $M=37,66$ & $M=43,06$ & $M=42,92$ & & $M=61,93$ & $M=43,25$ & $M=44,89$ & \\
\hline $45 a 64^{a}$ & $M=64,08$ & $M=58,78$ & $M=58,92$ & & $M=40,28$ & $M=58,60$ & $M=56,99$ & \\
\hline Est. civil: & $p=, 042$ & $p=, 045$ & \multirow{3}{*}{ n.s. } & $p=, 008$ & \multirow{3}{*}{ n.s. } & $p=, 051$ & \multirow{3}{*}{ n.s. } & $p=, 030$ \\
\hline Casado & $M=54,40$ & $M=54,11$ & & $M=55,34$ & & $M=54,29$ & & $M=54,50$ \\
\hline Não casad & $M=41,19$ & $M=42,04$ & & $M=38,48$ & & $M=41,52$ & & $M=40,90$ \\
\hline N. ensino: & $p=, 003$ & $p=, 057$ & $p=, 010$ & \multirow{3}{*}{ n.s. } & $p=, 057$ & \multirow{3}{*}{ n.s. } & \multirow{3}{*}{ n.s. } & $p=, 050$ \\
\hline Até $3^{\circ} \mathrm{cicl}$ & $M=57,69$ & $M=54,97$ & $M=56,47$ & & $M=46,64$ & & & $M=55,26$ \\
\hline Sec. e sup. & $M=40,36$ & $M=44,69$ & $M=42,31$ & & $M=57,94$ & & & $M=44,23$ \\
\hline Profissão: & $p=, 010$ & $p=, 033$ & \multirow{3}{*}{ n.s. } & \multirow{3}{*}{ n.s. } & $p=, 024$ & \multirow{3}{*}{ n.s. } & \multirow{3}{*}{ n.s. } & \multirow{3}{*}{ n.s. } \\
\hline Empregad & $M=43,28$ & $M=45,13$ & & & $M=57,85$ & & & \\
\hline Não empr. & $M=57,99$ & $M=56,32$ & & & $M=44,79$ & & & \\
\hline E. filhos: & $p=, 008$ & \multirow{3}{*}{ n.s. } & $p=, 028$ & $p=, 050$ & \multirow{3}{*}{ n.s. } & \multirow{3}{*}{ n.s. } & \multirow{3}{*}{ n.s. } & $p=, 008$ \\
\hline Com & $M=55,67$ & & $M=54,66$ & $M=54,35$ & & & & $M=55,47$ \\
\hline Sem & $M=38,82$ & & $M=41,46$ & $M=42,27$ & & & & $M=39,36$ \\
\hline
\end{tabular}


Note-se que, na escala que avalia qualidade de vida, para as cinco escalas de funcionalidade, valores altos significam uma baixa funcionalidade e, pelo contrário, valores baixos significam uma alta funcionalidade.

Deste modo, relativamente à variável idade, verificamos que os sujeitos com idades entre os 45 e os 64 anos a presentam níveis inferiores de funcionamento físico, de papel e cognitivo; uma percepção global de saúde e qualidade de vida inferiores; e, níveis superiores de fadiga e dor. Num estudo realizado por Gil-Fernandez et al. (2003), com a EORTC - OLQ-C30, as mulheres, sobreviventes a Linfoma de Hodgkin, com idade superior a 45 anos, apresentavam pior funcionamento físico; verificou-se, também, que os sujeitos sobreviventes com mais de 45 anos apresentavam um inferior estado de saúde e qualidade de vida globais, grande perda de a petite e, mais problemas de diarreia. Estes resultados são concordantes com os nossos no que diz respeito ao funcionamento físico e à saúde e qualidade de vida globais. Contudo, num estudo realizado por Persson, Larsson, Ohlsson e Hallberg (2001), não se encontraram diferenças significativas entre os grupos de idades estudadas, quando comparados sujeitos com Leucemia aguda e sujeitos com Linfoma maligno de alto grau. Estes resultados devem ser observados com moderação, visto que utilizam uma amostra muito reduzida e a metodologia não se inscreve totalmente na nossa.

Constatamos, no nosso estudo, que os sujeitos casados ou em união de facto apresentam níveis inferiores de funcionamento físico, de papel e social; apresentam, ainda, maiores dificuldades financeiras. Estes resultados podem estar relacionados com as exigências das tarefas características do casal, as quais dependem do estádio de desenvolvimento do ciclo vital da família. É compreensivel que os sujeitos a quem foram administradas altas doses de quimioterapia e, eventualmente, realizado auto-transplante de medula óssea, por terem a seu cargo uma família, uma casa, apresentem mais dificuldades físicas, tenham mais dificuldades no desempenho de papéis sociais, evidenciem maiores transtornos nas relações familiares e no meio social mais alargado, tal como na frequência de participação em actividades sociais e, tenham maiores dificuldades financeiras. Pelo que ficou descrito, consideramos de interesse investigar, futuramente, em que fase do ciclo de vida do casal se encontram os sujeitos casados da nossa amostra, com o intuito de melhor compreender os nossos resultados e, consequentemente, a forma de estar, agir e pensar dos sujeitos que vivenciaram uma doença hemato-oncológica.

Verificamos que os sujeitos que frequentaram até ao $3^{\circ}$ ciclo apresentam níveis inferiores de funcionamento físico e cognitivo e maiores dificuldades financeiras. Como possíveis justificações para os nossos resultados, consideramos que o facto dos sujeitos com menores habilitações académicas apresentarem pior funcio- 
namento físico, talvez se prenda com o desempenhar de funções de natureza mais física. Por outro lado, pensamos ser compreensível que quem tem uma literacia menor, apresente maiores dificuldades em lembrar-se das coisas ou em concentrar-se para ler ou ver televisão, devido a uma menor estimulação da actividade cognitiva. Por fim, se atendermos ao facto de que, geralmente, quem detém maiores habilitações académicas tem maiores rendimentos salariais, é compreensivel que quem frequentou o ensino secundário ou o ensino superior expresse menores dificuldades financeiras.

Para os sujeitos da nossa amostra, o envolvimento profissional parece conduzir a um melhor funcionamento físico e de papel, aumentando, também, a percepção de saúde e qualidade de vida globais, o que poderá ter repercussões ao nível de algumas dimensões do bem-estar, tal como anteriormente referimos.

A análise da variável existência de filhos revela que os sujeitos sem filhos apresentam uma maior funcionalidade física, cognitiva e social; e, ainda, menores dificuldades financeiras. No nosso entender, é compreensível que os sujeitos com filhos tenham despesas financeiras acrescidas, expressando maiores dificuldades neste campo. Como já defendemos, o diagnóstico de uma doença oncológica pode aumentar o sentido de alienação face à vida familiar e a perda de controlo das circunstâncias da vida, e a vivência desta doença, em que a morte é uma forte e real possibilidade e, a incerteza quanto à continuidade de uma vida familiar ou a antecipação de "perda" da família e dos filhos, pode conduzir a estes resultados.

Os sujeitos que realizaram transplante num período anterior até 24 meses relatam maiores dificuldades financeiras. Verificamos, então, que parte da hipótese 1 foi confirmada, no entanto, esta hipótese só é válida para as dificuldades financeiras, nos sujeitos auto-transplantados. Note-se que durante grande parte dos 24 meses, os sujeitos encontram-se em baixa clínica, sem poderem exercer as suas actividades profissionais e, com custos acrescidos devido à medicação que têm de tomar. A investigação realizada por Wettergren, Langius, Bjorkholm e Bjorvell (1997) revela que, no primeiro ano após auto-transplante, pode existir diminuição do funcionamento social, pela presença de dificuldades financeiras e de sintomas como a dispneia e a fadiga, resultados que são, parcialmente, concordantes com os nossos. Um estudo realizado por McQuellon et al. (1998) verificou que, um ano após realizar transplante de medula óssea, os níveis de energia apenas se encontravam a 70\%, embora a maioria dos sujeitos referissem satisfação com a sua qualidade de vida total. Outro estudo realizado por Broers, Kaptein, Cessie, Fibbe e Hengeveld (2000), permitiu concluir que, ao fim de três anos, um quarto dos sujeitos submetidos a TMO continuavam a relatar sérias limitações funcionais, tais como, perda de apetite, falta de energia e diminuição do interesse sexual. Também Saleh e Brockopp (2001), ao investigarem sujeitos com uma média de 30 
meses após $\mathrm{TMO}$, concluíram que aproximadamente $50 \%$ relataram moderados a severos problemas com a força e energia físicas.

Os resultados indicam que os sujeitos que entraram em remissão num período anterior até 24 meses apresentam níveis inferiores de funcionamento social. Concluise, então, que parte da hipótese 1 foi confirmada, no entanto, esta hipótese só é válida para o funcionamento social, nos sujeitos não transplantados. É no período após os tratamentos com altas doses de quimioterapia, que se tornam essenciais medidas rigorosas de auto-protecção com isolamento físico a nível social. Heutte et al. (2006), num estudo que realizou apenas com sobreviventes a Linfoma de Hodgkin, refere que a qualidade de vida melhora com o passar do tempo.

\subsection{Qualidade das relações familiares}

A análise da qualidade das relações familiares evidenciou que, de uma forma geral, para a população estudada, as áreas de maior fragilidade relacionam-se com a tentativa de manter a harmonia, escondendo os problemas ou mostrando poucas vezes que estão zangados. Estas atitudes podem traduzir-se numa qualidade relacional negativa, já que o "tranquilizar irrealista” ou a empatia excessiva podem não ser úteis ao serem percepcionados como minimizadores dos problemas, quando exprimidos por familiares e amigos (Helgeson e Cohen, 1999).

Por outro lado, as áreas de maior força são evidenciadas por: coesão (apoiar-se, relacionar-se, sentido de identidade, entreajuda, sentido de união e de poderem contar uns com os outros); baixo conflito (mínimo de agressões no campo físico, poucas zangas ou gritos, quase nunca perder a cabeça e não competir tentando ser um melhor que o outro); partilha (assuntos do dia-a-dia ou financeiros, problemas pessoais, conversar sobre vários assuntos, cuidado com o que dizem uns aos outros e liberdade de combinarem qualquer coisa em cima da hora). Perante uma situação de cancro, as condições fundamentais para o ajustamento individual e familiar são o elevado nível de coesão intra-familiar (Baider \& Kaplan De-Nour, 1986, 1988, 1996, referenciados por Pereira \& Lopes, 2005; Bloom, 1982, referenciados por Pereira \& Lopes, 2005; Friedman et al., 1988, referenciados por Pereira \& Lopes, 2005), a abertura e possibilidade de expressar os sentimentos (Holahan \& Moss, 1982, referenciados por Pereira \& Lopes, 2005; Moss, 1984, referenciados por Pereira \& Lopes, 2005) e a inexistência de conflitos intra-familiares (Spiegel, Bloom \& Gottheil, 1983, referenciados por Pereira \& Lopes, 2005). Alguns autores (Arpin et al., 1990, referenciados por Weihs \& Politi, 2005; Bloom, Pendergrass \& Burnell, 1984, referenciados por Weihs \& Politi, 2005; Lewis et al., 1989, referenciados por Weihs \& Politi, 2005) afirmam que um ambiente familiar coeso e com baixo conflito leva a que os seus membros tenham menos stress e melhores 
estratégias de adaptação. Outros (Giese-Davis, Hermanson, Koopman, Weibel \& Spiegel, 2000, referenciados por Weihs \& Politi, 2005; Kayser, Sormanti \& Straichamps, 1999, referenciados por Weihs \& Politi, 2005; Trask et al., 2003, referenciados por Weihs \& Politi, 2005) afirmam, ainda, que a alta expressividade emocional e a coesão das relações familiares prediz um melhor ajustamento em todos os membros familiares.

Assim, de acordo com a literatura, somos impelidos a deduzir que, de uma maneira geral, o clima relacional familiar dos participantes do nosso estudo constitui um recurso que poderá contribuir para níveis menos elevados de stress e utilização de estratégias mais adaptativas, ao lidarem com as questões relacionadas com a sua situação anterior de cancro. No entanto, como anteriormente referimos, a incerteza quanto à continuidade de vida ou a antecipação de morte podem catalisar uma certa alienação face à família, ou potenciar um sentido de angústia, receio, preocupação devido à possibilidade da perda de contacto com a família, diminuindo, assim, o impacto da positividade do clima relacional familiar. Contudo, não podemos confirmar ou infirmar as hipóteses 8 e 9, por nós propostas, visto que apenas fazemos uma análise qualitativa da dimensão Relação do questionário FES.

\subsection{Relação entre o sentido de coerência e o bem-estar psicológico}

Verificamos, através da análise de uma correlação de Spearman, que o SCO Global e a sua dimensão Avaliação e Expectativas sobre a Realidade correlacionam-se de forma significativa e positiva, com todas as escalas que avaliam o Bem-Estar Psicológico, inclusive com o BEP global, no entanto, com diferentes graus de intensidade (com valores que variam entre .211 e .584, $p<.05$ ou $p<.01$ ). Os valores mais fracos são os que se referem à associação do SCO Global com a dimensão Autonomia, e à associação de Avaliação e Expectativas sobre a Realidade com a dimensão Autonomia. A dimensão Percepção de Organização e Coerência apenas apresenta uma relação positiva e significativa com a dimensão aceitação de si (.320, p<.01). Estes resultados confirmam parte da hipótese 5 .

Atendendo ao modelo conceptual orientador do presente estudo, fica evidente que se o sujeito atribuir um significado (SCO) positivo à situação que expêriencia/ experienciou, a recuperação (BEP) tenderá a ser também positiva.

\subsection{Relação entre o sentido de coerência e a qualidade de vida}

Podemos afirmar, através de uma análise de uma correlação de Spearman, que os sujeitos da nossa amostra expressam que quanto maior o seu SCO global, maior 
o seu funcionamento físico, de papel, emocional, cognitivo e social; menor a sua fadiga, dor, dispneia e dificuldades financeiras; e maior a sua percepção de saúde e qualidade de vida globais, sendo o contrário também aplicável (os resultados para $p<.05$ ou $p<.01$, variam entre .204 e .363). Pensamos ser compreensível que, tal como evidenciam os nossos resultados, os sujeitos para atribuírem um significado positivo à situação que vivenciaram ou vivenciam (percepcionando-a como estruturada e explicável, reconhecendo os recursos e gerindo-os de acordo com as suas necessidades), apresentem um funcionamento positivo a vários níveis, poucos sintomas físicos, menores dificuldades financeiras e, avaliem a sua saúde e qualidade de vida globais de forma, também ela, positiva. Do mesmo modo que a existência de um bom funcionamento, poucos sintomas, menores dificuldades financeiras, e uma avaliação positiva da saúde e qualidade de vida globais, contribuem para aumentar o significado positivo dado à situação.

Relativamente à dimensão Avaliação e Expectativas sobre a Realidade, afirmamos que quanto mais positiva a avaliação do mundo e do próprio, bem como as expectativas sobre o próprio e sobre os outros, maior será o funcionamento físico, o emocional e o cognitivo; maior a saúde e a qualidade de vida globais; e, menor a fadiga, a dor e a dispneia, sendo o contrário também aplicável (os resultados para $p<.05$ ou $p<.01$, variam entre .213 e .327). No nosso entender, parece-nos plausível que o facto dos sujeitos avaliarem, de forma positiva, o mundo e o próprio e, apresentarem expectativas também positivas sobre o próprio e os outros, contribua para um bom funcionamento a vários níveis, poucos sintomas, e uma maior percepção de saúde e qualidade de vida globais.

No que diz respeito à dimensão Percepção de Organização e Coerência, os resultados mostram que quanto maior a percepção de organização e coerência do mundo e do próprio, maior será o funcionamento emocional e a percepção de saúde e qualidade de vida globais; e, menor a fadiga, a dor e as dificuldades financeiras, sendo o contrário também aplicável (os resultados para $p<.05$, variam entre .232 e .268). Pensamos ser compreensível que os sujeitos para percepcionarem, de forma positiva, a organização e coerência do mundo e deles próprios, o façam apoiados num bom funcionamento emocional, sintam poucos sintomas físicos e percepcionem uma boa saúde e qualidade de vida globais. Da mesma forma, pensamos ser compreensivel que sem estes indicadores positivos da qualidade de vida não seria evidente uma percepção positiva da organização e coerência do mundo e dos próprios sujeitos.

Pelos nossos resultados, acerca da relação entre o sentido de coerência e a qualidade de vida, podemos concluir que, na generalidade, foi confirmada a hipótese 3 e a hipótese 5 , por nós propostas. 


\subsection{Relação entre o bem-estar psicológico e a qualidade de vida}

Pela observação dos nossos resultados, através da análise de correlações de Spearman, verificamos que: a aceitação de si está associada positivamente a uma percepção favorável da sua saúde e qualidade de vida globais (.225, p<.05), e negativamente à presença de dispneia $(-.275, p<.01)$ e insónias $(-.197, p<.01)$; 0 crescimento pessoal está associado negativamente a uma avaliação positiva do funcionamento físico (-.269, p<.01), positivamente a saúde e qualidade de vida globais (.222, p<.05), e negativamente à presença de fadiga (-.211, p<.05); os objectivos na vida estão associados negativamente a funcionalidade (nomeadamente física (-.318, $p<.01)$, de papel $(-.238, p<.05)$ e cognitiva $(-.287, p<.01))$, positivamente a saúde e qualidade de vida globais $(.316, p<.01)$, negativamente a sintomas (tais como fadiga (-.247, p<.05), dor (-.254, p<.05), dispneia $(-.258, p<.01)$ e insónias $(-.215, p<.05))$, e negativamente a dificuldades financeiras (-.232, $p<.05)$; as relações positivas com os outros estão associadas negativamente a funcionamento físico (-.223, $p<.05)$ e cognitivo (-.284, $p<.01)$; positivamente a saúde e qualidade de vida globais (.239, $p<.01)$; e negativamente a fadiga (-.282, $p<.01)$, náuseas e vómitos (-.199, p<.05), dor (-.256, p<.01), dispneia (-.200, p<.05), insónias (-.221, p<.05) e perda de apetite (-.234, p<.05); o domínio do meio está associado positivamente a saúde e qualidade de vida globais (.200, p<.05); a autonomia está associada negativamente a funcionamento cognitivo $(-.306, p<.01)$ e social $(-.202, p<.05)$, e a dor $(-.207, p<.05)$;

Por último, o bem-estar psicológico global está associado negativamente a funcionalidade (essencialmente física (-.278, p<.01), cognitiva (-.314, p<.01) e social (-.208, p<.05)); positivamente a uma avaliação positiva da saúde e qualidade de vida globais (.306, p<.01); negativamente a sintomas (tais como fadiga (-.284, $p<.01)$, dor (-.304, $p<.01)$, dispneia $(-.220, p<.05)$ e insónias $(-.256, p<.01))$; e negativamente a dificuldades financeiras (-.198, $p<.05)$.

Novo (2003) referencia que os estados psicológicos positivos podem provocar efeitos positivos na saúde física. Também Ryff e Singer (1998) consideram que o que é positivo tem um importante efeito contra as emoções negativas e na promoção da saúde física, tal como, ter objectivos na vida, ter relações de qualidade com os outros, amor-próprio, auto-estima, auto-respeito, aceitação de si, sentido de eficácia, domínio e controle. Em analogia com o nosso estudo, consideramos que "os estados psicológicos positivos" são expressos pelo bem-estar psicológico global e suas dimensões, a "saúde física" é expressa pela qualidade de vida e os "efeitos positivos" referem-se às relações encontradas. 


\section{Reflexões Finais}

Com este estudo, a nossa finalidade é a de contribuir para o enriquecimento do conhecimento sobre o desenvolvimento de adultos que experienciaram uma doença hemato-oncológica, através de uma compreensão mais aprofundada de alguns factores que facilitam a sua adaptação, os quais podem ser considerados factores de resiliência - a percepção da sua situação de vida (sentido de coerência), os seus recursos familiares (qualidade das suas relações familiares) - e o seu aparente estado de adaptação actual (bem-estar psicológico e qualidade de vida).

Partindo das hipóteses prévias que colocámos, pretendemos, aqui, destacar as conclusões relativas aos resultados encontrados:

- Os sujeitos com um nível de escolaridade mais baixa percepcionam-se menos capazes de lidar com as exigências, de activar os recursos disponíveis e de implementar as estratégias de coping adaptativas.

- A submissão a auto-transplante de medula óssea parece ser um factor de stress que atinge, de forma significativa, comportamental e emocionalmente, psicológica e psicossomaticamente, os sujeitos da nossa amostra.

- Os sujeitos a quem foi diagnosticado Mieloma Múltiplo apresentam valores inferiores de: avaliação e expectativas sobre a realidade (percepção da situação), crescimento pessoal, objectivos na vida e BEP global (recuperação) - o que pode indicar que este diagnóstico seja indutor de maior stress.

- Relativamente à recuperação (BEP e QDV), os resultados parecem indicar que os participantes mais jovens da nossa amostra, os não casados, os com maior nível de ensino, os empregados e os que não têm filhos, têm uma maior predisposição para uma melhor recuperação.

- A análise qualitativa parece indicar que o clima relacional familiar constitui um recurso que poderá contribuir para níveis menos elevados de stress e utilização de estratégias mais adaptativas. Contudo, a incerteza quanto à continuidade de vida e a antecipação da morte podem catalizar uma certa alienação face à família, ou potenciar um sentido de angústia, receio ou preocupação devido à possibilidade de perda de contacto com a família, diminuindo, assim, o impacto da positividade do clima relacional familiar.

- A percepção da situação (SCO) correlaciona-se de forma positiva com a recuperação (BEP e ODV). Por sua vez, as variáveis que representam a recuperação também se correlacionam de forma positiva.

Consideramos importante salientar alguns dos aspectos menos positivos que, se ultrapassados, poderiam ter enriquecido a investigação: a dimensão limitada da 
amostra, constituída apenas pelos próprios doentes, o método de amostragem por conveniência, centrada exclusivamente num Hospital, algumas informações sócio-biográficas pouco detalhadas e o facto de alguns questionários se terem revelado insuficientemente ajustados às características específicas da nossa amostra (portadores de uma doença crónica grave).

Contudo, e apesar de todas as limitações, pensamos que este estudo pode contribuir para o desenrolar de futuras investigações. Assim, consideramos importante a sua continuação, numa investigação longitudinal. Os estudos longitudinais são uma ferramenta importante na compreensão não linear do desenvolvimento após uma situação de doença hemato-oncológica. Estes poderão, ainda, pensamos nós, ser um contributo essencial para a prevenção de processos negativos e a promoção dos processos positivos, de forma a potenciar ganhos em saúde.

Outra "continuidade", ainda, com o mesmo objectivo de contribuir para o aumento do conhecimento sobre esta temática e para potenciar os ganhos em saúde, constitui a realização de estudos desta natureza em outros locais a nível nacional, que englobassem amostras com idênticas características clínicas, e que envolvessem também elementos da família, com o intuito de poder efectuar-se um estudo comparativo de maior complexidade sistémica.

A realização deste estudo exploratório aponta, sobretudo, para a importância da continuidade de trabalhos nesta área já que vários poderão ser os benefícios para o exercício de Enfermagem. Em primeiro lugar, permitem enriquecer uma área de conhecimentos não muito desenvolvida a nível nacional, mas em progressiva ascensão. Por outro lado, um dos alicerces da prática de Enfermagem assenta na promoção da saúde. Pensamos que, com a realização desta investigação - onde se verificou existirem aspectos positivos na adaptação dos indivíduos após um acontecimento de vida gerador de grande stress - e o seu aprofundamento através de outros estudos, abrem-se portas para promover e reforçar esses aspectos e prevenir ou intervir mais precocemente perante aspectos que se revelem menos positivos. Não podemos deixar de mencionar a importância da necessidade de um acompanhamento psicológico, tanto dos indivíduos que vivenciaram uma doença hemato-oncológica, como dos seus familiares, também eles implicados neste processo. Realçamos, por exemplo, o facto de, neste estudo, termos verificado que, apesar do sistema familiar constituir um recurso bastante positivo no processo de adaptação - a avaliar pela boa qualidade das relações familiares na percepção dos sujeitos que vivenciaram a doença - existir um aparente "sub-aproveitamento" desse mesmo recurso. Isto porque os resultados que encontrámos, tal como referimos na discussão dos resultados, parecem indiciar uma minimização, ou mesmo anulamento, do impacto positivo da qualidade das relações familiares quer na percepção da situação, quer no bem-estar e qualidade 
de vida dos sujeitos. No entanto, sublinhamos a necessidade de estudos mais complexos que confirmem esta hipótese.

Salientamos, ainda, que, com o desenvolvimento da medicina, cada vez é maior o número de indivíduos que sobrevivem a uma doença oncológica. O caso específico das doenças hemato-oncológicas não é excepção. Assim, pensamos ser da competência e da responsabilidade dos enfermeiros estarem atentos e actualizados acerca das necessidades desta população e suas famílias, com o intuito de um acompanhamento sistemático e contínuo no campo da saúde.

\section{Referências bibliográficas}

Aaronson, N. K. et al. (1993). The european organization for research and treatment of cancer QLQ-C3O: a quality-of-life instrument for use in internacional clinical trials in oncology. Journal of the National Cancer Institute, 85(5), 365-376.

Antonovsky, A. (1998a). The sense of coherence - an historical and future perspective. In H. I. McCubbin, E. A. Thompson, A. I. Thompson \& J. E. Fromer (Ed.), Stress, coping and health in families - sense of coherence and resiliency (pp. 3-20). Thousand Oaks: Sage Publications.

Antonovsky, A. (1998b). The structure and properties of the sense of coherence scale. In H. I. McCubbin, E. A. Thompson, A. I. Thompson \& J. E. Fromer (Ed.), Stress, coping and health in families - sense of coherence and resiliency (pp. 21-40). Thousand Oaks: Sage Publications.

Baider, L., Koch, U., Esacson, R. \& De-Nour, A. K. (1998). Prospective study of cancer patients and their spouses: the weakness of marital strength. Psycho-oncology, 7, 49-56.

Boyle, D. A. (2006). Survivorship. Clinical journal of oncology nursing, 10, 407-416.

Broers, S., Kaptein, A. A., Cessei, S. L., Fibbe, W. \& Hengeveld, M.W. (2000). Psychological functioning and quality of life following bone marrow transplantation: a 3-year follow-up study. Journal of Psychosomatic Research, 48, 11-21.

Gil-Fernández, J. J. et al. (2003). Quality of life and psychological well-being in Spanish long-term survivors of Hodgkin's disease: results of a controlled pilot study. Annual Hematology, 82, 14-18.

Haas, B. K. (1999). A multidisciplinary concept analysis of quality of life. Western Journal of Nursing Research, 21, 728-742.

Helgeson, V. S. \& Cohen, S. (1999). Social support and adjustment to cancer: reconciling descriptive, correlational, and intervention research. In R. M. Suinn \& G. R. VandenBos (Eds.), Cancer patients and their families: readings on disease course, coping, and psychological interventions (pp. 53-79). Washington, DC: American Psychological Association.

Heutte, N. et al. (2006, Junho). Results of a longitudinal survey on quality of life (QoL) in 935 patients with supradiaphragmatic early stage Hodgkin lymphoma $(\mathrm{HL})$ enrolled in the EORTC-GELA H8trial (\# 20931). Poster apresentado no ASCO Meeting, Atlanta, Georgia - US. 
Holmberg, S., Thelin, A. \& Stiernstrom, E. (2004). Relationship of sense of coherence to other psychosocial indices. European Journal of Psychological Assessment, 20, 227-236.

Kralik, D. (2002). The quest for ordinariness: transition experienced by midlife women living with chronic illness. Journal of Advanced Nursing, 39, 146-154.

Kornblith, A. B. et al. (1992). Hodgkin disease survivors at increased risk for problems in psychosocial adaptation. Cancer, 70, 2214-2224.

Levinson, D. (1990). A theory of life structure development in adulthood. In C. Alexander, \& E. Lander (Eds.), Higher stages of human development (pp.35-53). Oxford: Oxford University Press.

Marchand, H. (2001). Temas de desenvolvimento psicológico do adulto e do idoso. Coimbra: Quarteto.

McQuellon, R. P., Russell, G. B., Rambo, T. D., Craven, B. L., Radford, J., Perry, P. P. \& Cruz, J. (1998). Quality of life and psychological distress of bone marrow transplant recipients: the 'time trajectory' to recovery over the first year. Bone Marrow Transplantation, 21, 477-486.

Moos, R. H. (1990). Conceptual and empirical approaches to developing family-based assessment procedures: resolving the case of family environment scale. Family Process, 29, 199-208.

Moura, O. (2005). Avinculação aos pais e relações familiares na adolescência. Dissertação de Mestrado em Psicologia. Faculdade de Psicologia e de Ciências da Educação da Universidade do Porto.

Nilsson, B., Holmgren, L. \& Westman, G. (2000). Sense of coherence in different stages of health and disease in northern Sweden - gender and psychosocial differences. Scandinavian Journal of Primary Health Care, 18, 14-20.

Novo, R. F. (2003). Para além da eudaimonia: o bem-estar psicológico em mulheres na idade adulta avançada. Lisboa: Fundação Calouste Gulbenkian: Fundação para a Ciência e a Tecnologia. Ministério da Ciência e do Ensino Superior.

Pereira, M. G. \& Lopes, C. (2005). O doente oncológico e a sua familia ( $2^{\mathrm{a}}$ ed.). Lisboa: Climepsi Editores.

Persson, L., Larsson, G., Ohlsson O. \& Hallberg I. R. (2001). Acute leukaemia or highly malignant lymphoma patients' quality of life over two years: a pilot study. European Journal of Cancer Care, 10, 36-47.

Ribeiro, J. L. P. (1998). Psicologia e Saúde. Lisboa: Instituto Superior de Psicologia Aplicada.

Ribeiro, J. L. P. (2001). Qualidade de vida e doença oncológica. In M. R. Dias \& E. Durà (Eds.), Territórios da psicologia oncológica (pp. 75-98). Lisboa: Climepsi Editores.

Ribeiro, J. L. P. (2006). Relação entre a psicologia positiva e as suas variáveis protectoras e a qualidade de vida e bem-estar como variáveis de resultado. In I. Leal (Ed.), Perspectivas em psicologia da saúde (pp. 231-244). Coimbra: Quarteto.

Riley, J. R. \& Masten, A. S. (2005). Resilience in context. In R. V. Peters, B. Leadbeater \& R. J. McMahon (Eds.), Resilience in children, families, and communities: linking context to practice and policy (pp. 13-25). New York: Kluwer Academic / Plenum Publishers.

Rolland, J. S. (1989). Chronic illness and the family life cycle. In B. Carter \& M. McGoldrick (Eds.), The changing family life cycle - a framework for family ( $2^{\mathrm{a}}$ ed., pp. 433-456). Needham Heights: Prentice Hall College Div.

Ryff, C. D. (1989). Happiness is everything, or is it? Explorations on the meaning of psychological well-being. Journal of Personality and Social Psychology, 57, 1069-1081. 
Ryff, C. D. (1991). Possible selves in adulthood and old age: a tale of shifting horizons. Psychology and Aging, 6, 286-295.

Ryff, C. D. \& Keyes, C. L. M. (1995). The structure of psychological well-being revisited. Journal of Personality and Social Psychology, 69, 719-727.

Ryff, C. D. \& Singer, B. (1998). The contours of positive human health. Psychological Inquiry, 9, 1-28.

Saleh, U. S. \& Brockopp, D. Y. (2001). Quality of life one year following bone marrow transplantation: psychometric evaluation of the quality of life in bone marrow transplant survivors tool. Oncology Nursing Forum, 28, 1457-1464.

Santos, C. S. V. B. \& Ribeiro, J. L. P. (2001). A qualidade de vida do doente oncológico - revisão da bibliografia. Referência, 7, 5-16.

Seligman, M. E. P. \& Csikszentmihalyi, M. (2000). Positive psychology: an introduction. American Psychologist, 55, 5-14.

Svartik, L., Lidfeldt, J., Nerbrand, C., Samsioe, G., Schersten, B. \& Nilsson, P. M. (2000). Dyslipidemia and impaired well-being in middle-aged women reporting low sense of coherence - the Women's Health in the Lund Area (WHLA) study. Scandinavian Journal of Primary Health Care, 18, 177-182.

Walsh, F. (1998). Strengthening family resilience. New York: The Guilford Press.

Wettergren, L., Langius, A., Bjorkholm, M. \& Bjorvell, H. (1997). Physical and psychosocial functioning in patients undergoing autologous bone marrow transplantation - a prospective study. Bone Marrow Transplantation, 20, 497-502.

Wettergren, L., Langius, A., Bjorkholm, M. \& Bjorvell, H. (1999). Post-traumatic stress symptoms in patients undergoing autologous stem cell transplantation. Acta Oncologica, 38, $475-480$.

Weihs, K. \& Politi, M. (2005). Family development in the face of cancer. In D. R. Crane \& E. S. Marshall (Eds.), Handbook of families \& health: interdisciplinary perspectives (pp. 3-18). Thousand Oaks: Sage Publications.

World Health Organization (1997). WHOOOL - Measuring quality of life. Retirado em Setembro 26, 2006, de http://www.who.int/mental_health/media/68.pdf

\section{Factors for adaptation and recovery after a haemato-oncologic disease - an exploratory study}

We intend to analyze factors that can favour or make it difficult the process of adaptation in individuals with a haemato-oncologic disease. The data gathering, with a sample of 101 individuals in situation of remission of a haemato-oncological disease, included the social-biographical questionnaire, the coherence sense questionnaire - Questionnaire of Orientation for Living (QOV - Antonovsky, 1998b), the Psychological Wellbeing Questionnaire (BEP - Ryff, 1989), the Quality of Life Questionnaire (EORTC OLQ-C3O - version 3- Aaronson et al., 1993) and the Family Relations Questionnaire (FES- Moos, 1990). The results obtained allow us to realise that the subjects with a lower teaching level attended are perception less capable to adapt of stress; the submission to autologous bone marrow transplantation 
is additional and significant factor of stress; the diagnosis of Multiple Mieloma seems to be inductive of greater stress; youngest, not married, with high school or graduation, employees and without offspring have greater predisposition for one better recovery; and, the family relational climate constitutes a resource that may contribute for lower levels of stress and use of more fitted strategies.

KEY-WORDS: Haemato-oncologic diseases, sense of coherence, psychological wellbeing, quality of life, familiar relational climate.

\section{Facteurs d'adaptation et de recuperation apres une maladie hémato- oncologique - une étude exploratoire}

Nous prétendons analyser des facteurs qui peuvent favoriser ou difficulter le processus d'adaptation chez des personnes avec une maladie hémato-oncologique. La collecte de données, avec un échantillon de 101 sujets en rémission d'une maladie hémato-oncologique, a inclu un questionnaire socio biographique, le Questionnaire d' Orientation Pour Vivre (QOV- Antonovsky, 1998b), le Questionnaire de Bien-être Psychologique (BEP- Ryff, 1989), le Questionnaire de la Qualité de Vie (EORTC OLQC30 - version 3 - Aaronson et al., 1993) et, le Questionnaire de la Qualité des Relations Familières (FOIS Moos, 1990). Les résultats obtenus nous permettent de constater que les sujets avec un niveau de scolarité plus bas se perceptionnent moins capables de s'adapter au stress; l' auto-transplant de moelle osseuse est un facteur de stress aditionel et significatif; le diagnostique de Miéloma Multiple semble induir un plus grand stress; les plus jeunes, non mariés, avec plus grand niveau de scolarité, les employés et ceux qui n'ont pas d' enfants ont une plus grande prédisposition pour une meilleure récupération ; et, le climat relationnel familié constitue une ressource qui pourra contribuer pour des niveaux moins élevés de stress et à l' utilisation de stratégies plus adaptatives.

MOTS-CLÉS: Maladies hémato-oncologiques, sens de cohérence, bien-être psychologique, qualité de vie, climat relationnel familié. 